\title{
Proposta de Tabela de Medidas Associáveis para uso no vestuário feminino a partir dos sete biótipos brasileiros
}

\author{
Proposal of a Table of Associable Body Measurements for use in women's apparel \\ from the seven Brazilian biotypes
}

\author{
LONGHI, Tatiana; Mestra; Universidade do Estado de Santa Catarina - UDESC \\ dilonghi@gmail.com
}

SANTOS, Flávio Anthero Nunes Vianna dos; Doutor; Universidade do Estado de Santa Catarina UDESC

flavioanvs@hotmail.com

\begin{abstract}
Resumo
Um estudo recente, realizado em nível nacional, confirmou a existência de sete biótipos na população feminina brasileira. Este artigo é parte dos resultados obtidos em pesquisa de mestrado, cujo objetivo foi o estudo dos biótipos femininos, bem como os algoritmos que os definem. De posse destes, foi possível desenvolver uma Tabela de Medidas Associáveis (TMA), na qual constam medidas de sete biótipos, além da possibilidade de combinação com três estaturas. Para que os valores pudessem ser definidos corretamente, desenvolveu-se um software para o cálculo dos valores de cada biótipo - a Calculadora de Biótipos. A TMA permite ao modelista trabalhar com os dados de mais de um biótipo simultaneamente e utilizar comprimentos variados, além de permitir acrescentar dados de outros biótipos ou retirar aqueles que não tenham representatividade.
\end{abstract}

Palavras Chave: biótipos; tabela de medidas; modelagem.

\begin{abstract}
A recent study, conducted at the national level, confirmed the existence of seven biotypes in the Brazilian female population. This article is part of the results obtained in master's research, whose objective was the study of the female biotypes, as well as the algorithms that define them. With this in mind, it was possible to develop a Table of Associable Body Measurements, which includes measurements of seven biotypes, besides the possibility of combining with three statures. In order for the values to be correctly defined, a software was developed to calculate the values of each biotype - the Biotype Calculator. The TMA allows the modeler to work with data from more than one biotype simultaneously and use varied lengths, in addition to allowing the addition of data from other biotypes or removing those that are not representative.
\end{abstract}

Keywords: biotypes; table of body measurements; patternmaking. 


\section{Introdução}

O vestuário é um dos bens de consumo que acompanha o ser humano desde o início da civilização até os dias atuais e como tal, evoluiu ao ponto de ajustar-se ao corpo humano como uma segunda pele. Esse resultado pode ser alcançado por meio da tecnologia dos materiais têxteis e também da modelagem do vestuário. Esta última estuda as formas e os movimentos das pessoas a fim de produzir vestuário que além da função estética e social, também permita a manutenção da saúde e a liberdade dos movimentos.

De acordo com Dinis e Vasconcelos (2014), além da expressão criativa e de uma metodologia de trabalho, a modelagem do vestuário exige precisão matemática. A ferramenta que fornece dados matematicamente precisos acerca das medidas do corpo humano é a tabela de medidas do vestuário. As tabelas são exclusivas de um determinado público - feminino, masculino ou infantil e podem ser mais específicas, identificando inclusive a faixa etária a que se destinam, como por exemplo, mulher jovem, mulher madura etc. A definição dos valores das medidas segue regras preestabelecidas pelo setor de vestuário e atendem a uma proporção fixa, que conforme vai progredindo numericamente, vai abrangendo do menor ao maior usuário. Segundo Jones (2005), às vezes essas medidas baseiam-se em pesquisas com consumidores, mas frequentemente são utilizados dados demográficos estimados empiricamente ou idealizados.

No Brasil não há um padrão de dimensionamento feminino referencial vigente, em função do cancelamento em 2012, da NBR 13377 (Medidas do corpo humano para vestuário - padrões referenciais). A norma era fornecida pela $A B N T^{1}$ como padrão de medidas femininas e foi extinta por não representar a realidade dos variados biótipos encontrados nas diversas regiões brasileiras. (ABNT, 2014).

Publicações internacionais, de países que realizaram pesquisas antropométricas da sua população, como os Estados Unidos, por exemplo, possuem mais de uma tabela de medidas, em função dos diferentes biótipos encontrados em sua população. Já as tabelas utilizadas no Brasil, contemplam somente o biótipo ideal, cujas medidas principais apresentam proporcionalidade estética e não indicam nenhuma parte do corpo que destoe de uma determinada proporção numérica de medidas.

A falta de padrões de medidas causa confusão e perdas, especialmente para a usuária do sexo feminino, que acaba adquirindo peças sem o formato mais adequado para o seu corpo. Essa inadequação também gera frustração às usuárias que acreditam estarem fora do padrão estético vigente por não encontrarem opções disponíveis que às atendam adequadamente. (KEISER e GARNER, 2008).

Um problema comum às tabelas de medidas encontradas em diversas publicações da área, é que estas se baseiam em um único biótipo e a partir dele desenvolvem sua numeração. Entretanto, em função da grande diversidade étnica, novos hábitos alimentares, atividade física e demais fatores, essas medidas encontram-se desatualizadas.

No âmbito industrial, segundo Dinis e Vasconcelos (2014), são utilizados os mais variados

\footnotetext{
1 A Associação Brasileira de Normas Técnicas, através das NBRs - Normas Brasileiras Regulamentadoras estabelece padrões voluntários de regulamentação. Estão em vigor atualmente a NBR 16060 Vestibilidade para homens corpo tipo normal, atlético e especial e a NBR 15800 Vestibilidade de roupas para bebê e infanto-juvenis.
} 
recursos no desenvolvimento das tabelas de medidas, tais como cópia de outras empresas ou o uso de medidas baseadas em normas técnicas que já se encontram ultrapassadas. Entretanto, as tabelas de medidas, além de desatualizadas são incompletas e, muitas vezes, estruturadas de forma simplificada, pois se considera que servem somente como um "modelo" de diretriz a ser seguida para o desenvolvimento dos diagramas que originam os moldes.

A utilização de uma tabela baseada em um único biótipo e que desconsidera a estatura dos indivíduos, resulta invariavelmente em um molde que originará uma peça que só será adequada a um tipo físico, relegando aos demais, incômodos como folgas desnecessárias e comprimentos inadequados. Isso porque, dentro dessa lógica de uma tabela tão simples, o fator preponderante é o peso corporal e não o formato do corpo, ou seja, uma pessoa escolhe a sua roupa em função da totalidade do seu tamanho e não em função da sua forma. Esse procedimento é totalmente contrário ao conceito de ergonomia e fatores humanos, uma vez que o produto é que deve se ajustar ao usuário é não o contrário.

Em função da falta de padrões referenciais de medidas e da falta do conhecimento acerca dos biótipos da população, Universidades e Escolas de Moda adotam padrões de medidas encontrados em fontes bibliográficas. No que tange à transmissão do conhecimento em modelagem do vestuário, essa simplificação da tabela de medidas traz uma série de prejuízos. Gera um impacto menor para o profissional experiente por ser complementado pelo conhecimento empírico, mas para um estudante que começa a conhecer a disciplina de modelagem, pode favorecer um aprendizado inconsistente. Isso porque, ao se deparar com dados tão simplificados acerca do corpo humano, esse aluno ignora toda a complexidade do projeto de produto cujo foco é o ser humano, com toda a sua diversidade. Posteriormente, esse estudante que se tornará um profissional, poderá vir a perpetuar a cultura de que o produto industrial de vestuário possui limitações em função da escala, e de que cabe ao consumidor compreender essas limitações e pagar por alterações que julgue necessárias ao ajuste do seu corpo.

Pesquisas têm revelado que pessoas "fora do padrão ideal" são a regra e não a exceção. De acordo com Keiser e Garner (2008), dados do SizeUSA (estudo antropométrico norte-americano) revelaram ser a forma retangular a mais comumente encontrada entre a população feminina norte-americana. Segundo as autoras, boa parte das indústrias vinha baseando sua produção de vestuário no formato ampulheta, considerado como padrão. Pesquisas de campo realizadas diretamente com os usuários, fornecem dados atualizados acerca dos atuais padrões corporais das pessoas e podem servir para uma reformulação nos padrões atuais de medidas.

\section{Identificação matemática dos biótipos femininos}

O conceito de biótipo deriva da classificação dos seres humanos em tipos em função das suas características morfológicas corporais. Existem diversas classificações, porém a adotada aqui vem a ser a mesma classificação utilizadas nos estudos que levaram a realização do SizeBR.

Os algoritmos de definição dos biótipos são provenientes de criteriosa análise de todos os estudos anteriores feitos em somatotipia, medição 3D e do trabalho de pesquisadores que há mais de trinta anos atuam na área do vestuário. Assim, a partir das pesquisas de doutorado de Carla Simmons, a equipe de Simmons, Stook e Devarajan (2004) desenvolveu o software Female Figure Identification Technique (FFIT) for Apparel ${ }^{\mathcal{O}}$. 
Nesse estudo, foram feitas medições de participantes em body scanner 3D, a partir das quais foi possível estabelecer os grupos com formas similares, resultando em nove biótipos identificados e caracterizados. São eles: Hourglass, Bottom Hourglass, Top Hourglass, Spoon, Triangle, Inverted Triangle, Rectangle, Oval e Diamond.

Lee et al. (2007), por sua vez, optou por apresentadas as fórmulas matemáticas ou algoritmos de sete dos nove biótipos estudados - Hourglass, Bottom Hourglass, Top Hourglass, Spoon, Triangle, Inverted Triangle e Rectangle (Figura 1).

Figura 1 - Os sete biótipos femininos

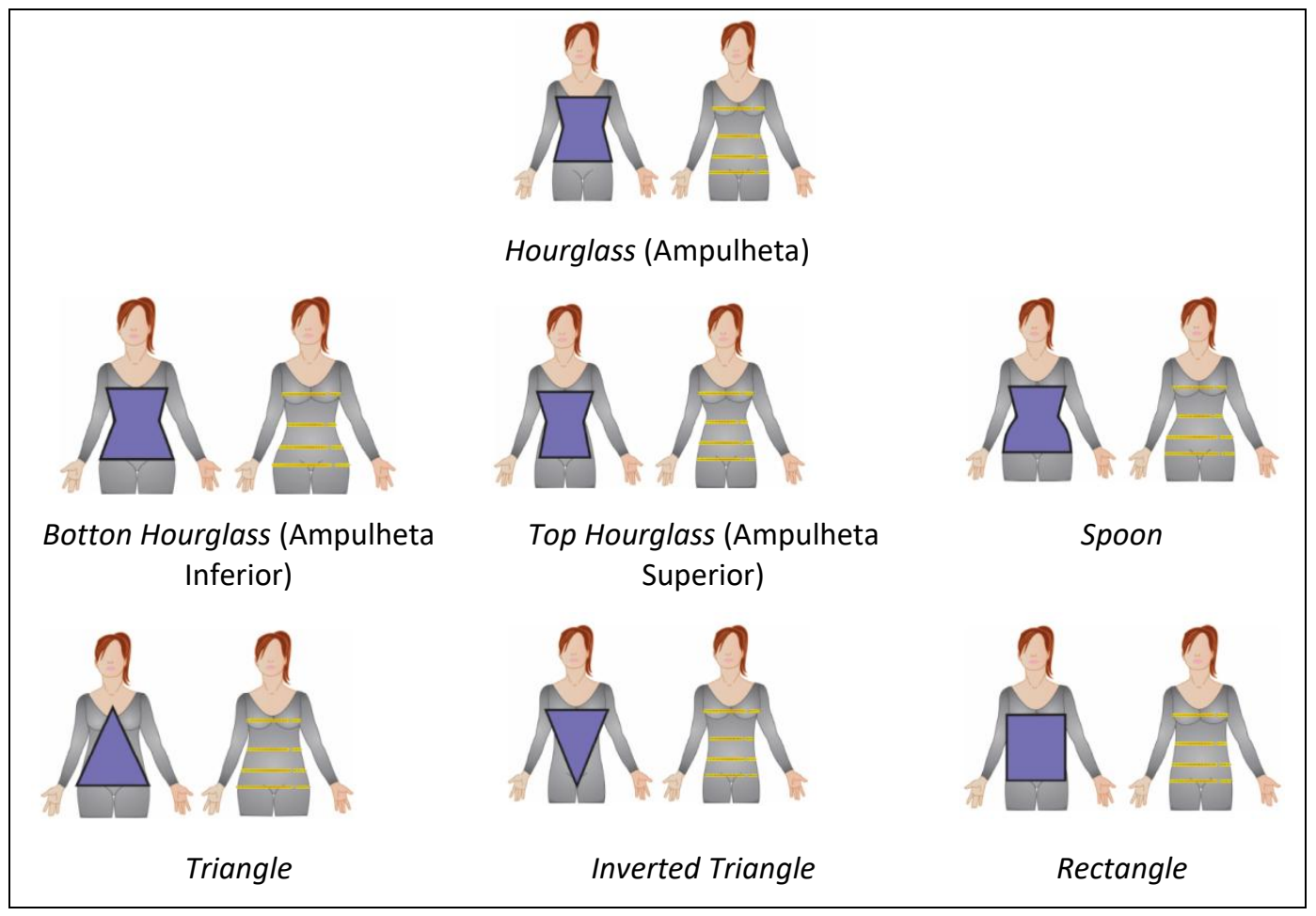

Fonte: adaptado de Bastos e Sabrá (2014)

Para definir a forma desses corpos, foram usadas quatro medidas corporais: waist circumference, hips circumference, high hip circumference e bust circumference. Os algoritmos gerados são apresentados no quadro 1.

Quadro 1 - Algoritmos de definição dos biótipos de Lee et al.(2007)

\begin{tabular}{|c|c|}
\hline Ampulheta & Ampulheta Inferior \\
\hline $\begin{array}{l}\text { If (bust-hips) }<=1 * 2.54 \text { then } \\
\text { If (hips-bust) }<3.6 * 2.54 \text { then } \\
\text { If (bust-waist) }>=9 * 2.54 \text { or (hips-waist) >= } \\
10 * 2.54 \text { then } \\
\text { shape }=\text { "Hourglass" }\end{array}$ & $\begin{array}{l}\text { If (hips-bust) }>=3.6 * 2,54 \text { and (hips-bust) }<10 \\
* 2.54 \text { then } \\
\text { If (hips-waist) }>=9 * 2.54 \text { then } \\
\text { If (high hip/waist) }<1,193 \text { then } \\
\text { shape }=\text { "Bottom Hourglass" }\end{array}$ \\
\hline Ampulheta Superior & Colher \\
\hline $\begin{array}{l}\text { If (bust-hips) }>1 * 2.54 \text { and (bust-hips) }<10 * 2.54 \\
\text { then }\end{array}$ & $\begin{array}{l}\text { If (hips-bust) }>2 * 2.54 \text { then } \\
\text { If (hips-waist) }>=7 * 2.54 \text { then }\end{array}$ \\
\hline
\end{tabular}




\begin{tabular}{|c|c|}
\hline $\begin{array}{l}\text { If (bust-waist) > = 9*2.54 then } \\
\text { shape }=\text { "Top Hourglass" }\end{array}$ & $\begin{array}{l}\text { If (high hip/waist) > = } 1.193 \text { then } \\
\text { shape = "Spoon" }\end{array}$ \\
\hline Triângulo & Triângulo Invertido \\
\hline $\begin{array}{l}\text { If (hips-bust) }>=3.6 * 2.54 \text { then } \\
\text { If (hips-waist) }<9 * 2,54 \text { then } \\
\text { shape }=\text { "Triangle" }\end{array}$ & $\begin{array}{l}\text { If (bust-hips) }>=3.6 * 2.54 \text { then } \\
\text { If (bust-waist) }<9 * 2.54 \text { then } \\
\text { shape }=\text { "Inverted Triangle" }\end{array}$ \\
\hline Retângulo & \\
\hline $\begin{array}{l}\text { If (hips-bust) }<3.6 * 2.54 \text { and (bust-hips) }<3.6 * 2.54 \\
\text { then } \\
\text { If (bust-waist) }<9 * 2.54 \text { and (hips-waist) }< \\
10 * 2.54 \text { then } \\
\text { shape = "Rectangle" }\end{array}$ & \\
\hline
\end{tabular}

Fonte: adaptado de Lee et al. (2007)

A pesquisa conduzida por Lee et al. (2007), fez uma comparação entre o corpo de norteamericanas e coreanas. Para isso, foram utilizadas as fórmulas dos sete biótipos anteriormente apresentados e body scanner 3D. As imagens a seguir mostram exemplos de mulheres com cada um dos sete biótipos estudados.

O biótipo ampulheta (hourglass), exemplificado na figura 2, apresenta uma pequena diferença entre as circunferências do busto e do quadril, além disso, as razões busto-cintura e quadril-cintura são praticamente iguais e significativas. A mulher com o biótipo ampulheta aparenta ser proporcional no busto e no quadril, com uma cintura bem marcada. (BASTOS e SABRÁ, 2014).

Figura 2 - Exemplo de mulheres com corpo em formato ampulheta

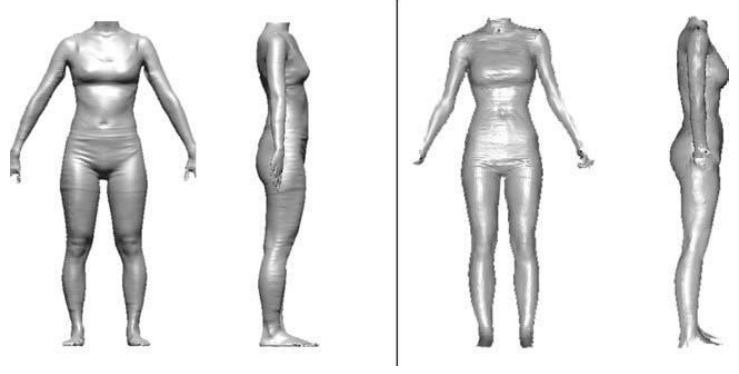

Fonte: Lee et al. (2007)

O biótipo ampulheta inferior (bottom hourglass), exemplificado na figura 3, assim como o biótipo ampulheta, é definido a partir das circunferências do busto, da cintura e do quadril, além da circunferência do quadril alto, sendo o quadril maior do que o busto. As razões busto-cintura e quadril-cintura são suficientemente significativas para produzir uma cintura marcada. (BASTOS e SABRÁ, 2014). 
Figura 3 - Exemplo de mulheres com corpo em formato ampulheta inferior
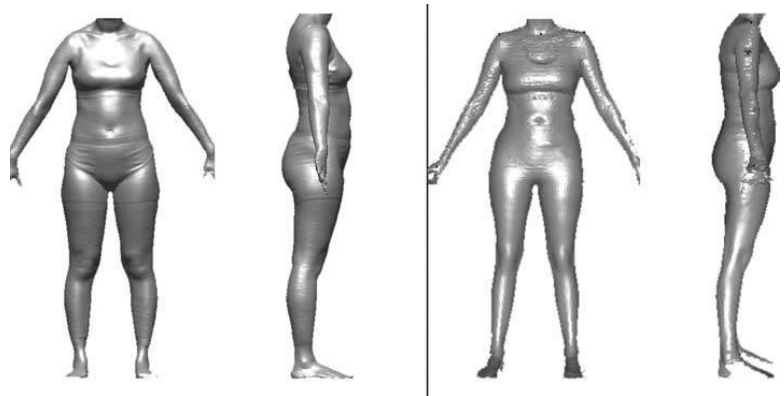

Fonte: Lee et al. (2007)

O biótipo ampulheta superior (top hourglass), exemplificado na figura 4, tem a circunferência do busto maior que a circunferência do quadril e razões busto-cintura e quadrilcintura, significativas, produzindo uma cintura marcada. (BASTOS e SABRÁ, 2014).

Figura 4 - Exemplo de mulheres com corpo em formato ampulheta superior
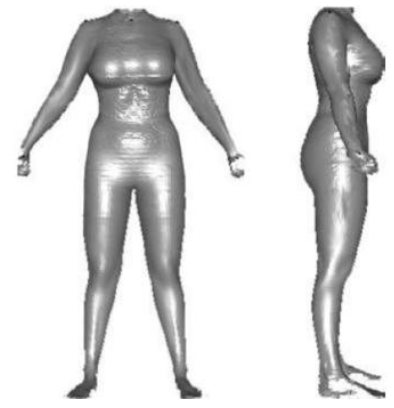

Fonte: Lee et al. (2007)

O biótipo colher (spoon), exemplificado na figura 5 , é determinado utilizando as circunferências do tórax, da cintura, do quadril e do quadril alto. Este biótipo é definido quando há uma diferença positiva entre as circunferências do quadril e do busto. Apresenta também razão busto-cintura menor que a do biótipo ampulheta e um valor alto na razão quadril alto-cintura. (BASTOS e SABRÁ, 2014).

Figura 5 - Exemplo de mulheres com corpo em formato colher

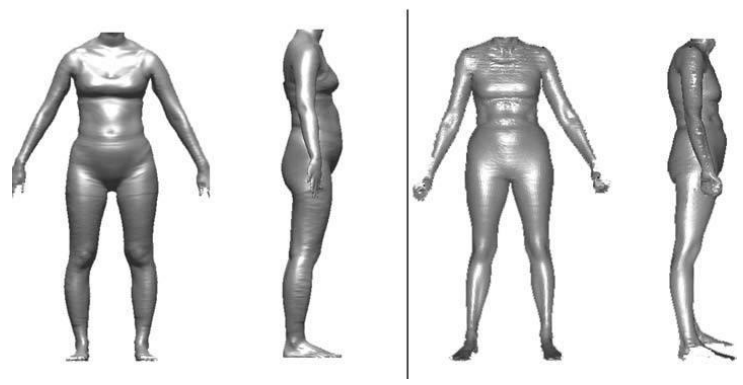

Fonte: Lee et al. (2007)

No biótipo triângulo (triangle), exemplificado na figura 6, a circunferência do quadril é 13 Congresso Brasileiro de Pesquisa e Desenvolvimento em Design, Univille, Joinville (SC) 
maior que a circunferência do busto e a razão quadril-cintura é pequena. Nesse biótipo o quadril é bem maior que o busto e a cintura tende a ser mais reta. Este biótipo é diferente do biótipo ampulheta inferior, pois não leva em consideração a razão busto-cintura. (BASTOS e SABRÁ, 2014).

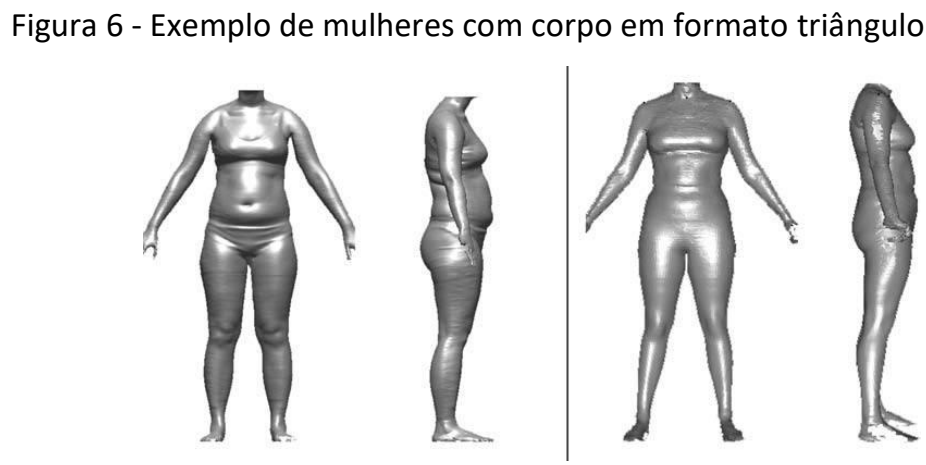

Fonte: Lee et al. (2007)

No biótipo triângulo invertido (inverted triangle), exemplificado na figura 7, a circunferência do busto é maior que a circunferência do quadril e a razão busto-cintura é pequena. O biótipo triângulo invertido não apresenta uma cintura marcada e não considera a razão quadril-cintura como é considerado no biótipo ampulheta superior. (BASTOS e SABRÁ, 2014).

Figura 7 - Exemplo de mulheres com corpo em formato triângulo invertido
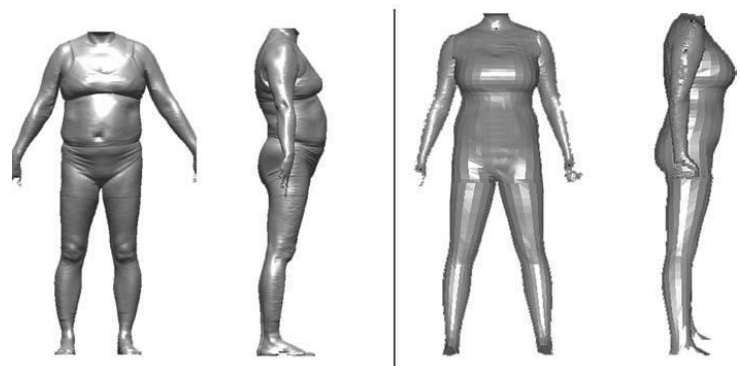

Fonte: Lee et al. (2007)

No biótipo retângulo (rectangle), exemplificado na figura 8, as circunferências do busto e do quadril são aproximadamente iguais. Apresenta valores baixos nas razões busto-cintura e quadril-cintura e uma linha de cintura não muito marcada. Portanto, o busto, a cintura e o quadril estão alinhados uns com os outros. (BASTOS e SABRÁ, 2014). 
Figura 8 - Exemplo de mulheres com corpo em formato retângulo
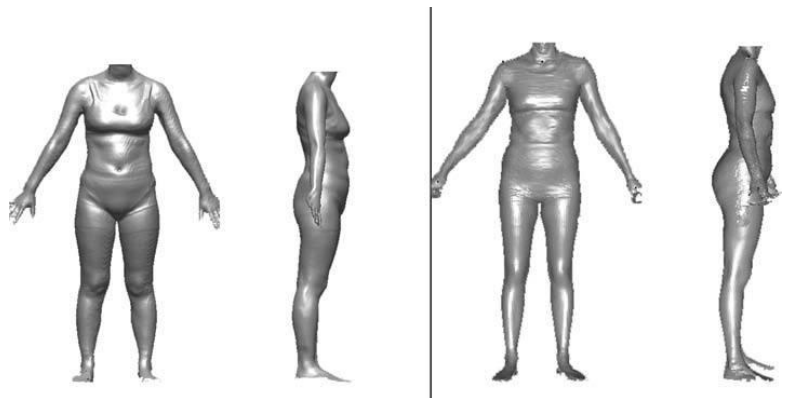

Fonte: Lee et al. (2007)

De acordo com Bastos e Sabrá (2014), a mulher brasileira em sua maioria, tem o corpo na forma retangular, especialmente na região nordeste com $70,3 \%$. Por meio da distribuição das formas ao longo da faixa etária pode-se inferir que conforme a mulher brasileira vai envelhecendo, seu corpo vai se aproximando cada vez mais da forma retangular. Como a amostragem não é estratificada, o quantitativo de mulheres mais velhas não tem significância estatística para representar toda a população brasileira de mulheres de mais de 56 anos. 0 formato ampulheta, por sua vez, embora amplamente utilizado como padrão estético no Brasil e no mundo, tem pouca representatividade numérica.

\section{Materiais e métodos}

O estudo é fruto da pesquisa de mestrado de Longhi (2017) e divide-se em dois momentos: o desenvolvimento de uma tabela de medidas associáveis com dados dos sete biótipos pesquisados e a pesquisa de campo. Este artigo aborda a primeira parte do estudo, na qual a partir dos algoritmos dos biótipos, foi possível definir os materiais e os métodos para a construção da TMA

Além do material teórico de definição dos sete biótipos femininos, que vêm a ser os algoritmos descritos por Lee et al. (2007), para o preenchimento dos valores referentes a cada medida de cada um dos biótipos, seria necessário alguns dados básicos. Como referência inicial de tabela de medidas femininas, foi utilizada a Tabela de Medidas do Curso de Moda da UDESC. A partir da definição do tamanho 38 conforme os números da referida tabela, seria possível preencher todos os dados do biótipo ampulheta neste tamanho inicial. Com uma progressão aritmética de quatro centímetros, foi possível preencher todos os demais números. Porém ainda seria recomendável a verificação do enquadramento dos valores dentro do algoritmo do biótipo ampulheta.

Também surgiu a necessidade de verificação da validade dos valores dos demais biótipos em função de seus respectivos algoritmos, pois somente depois dessa conferência seria possível definir os demais valores da tabela. Para tal, foi desenvolvido por Longhi e Smiderle (2016) um software para verificação de biótipos, o qual após a inserção das medidas gera como saída um dos sete biótipos. De posse desses materiais iniciou-se a construção da TMA. 


\subsection{Software para identificação dos biótipos}

As tabelas de medidas pesquisadas no presente estudo apresentam os dados de uma única combinação numérica e frequentemente não apresentam embasamento para as medidas que nelas constam, uma vez que são criadas através do conhecimento empírico. Com as pesquisas antropométricas que vêm sendo desenvolvidas, foram apresentados os algoritmos que definem as combinações numéricas que representam as diversas formas físicas femininas.

Através da pesquisa que conduziu aos estudos de Simmons, Stook e Devarajan (2004) e de posse dos algoritmos de Lee et al. (2007) tornou-se possível calcular valores que pudessem compor uma tabela de medidas. Porém, esse processo feito manualmente seria demorado e suscetível a erros de cálculo. Diante dessa questão, optou-se por desenvolver um software para cálculo dos biótipos, de modo a facilitar o processo. O programa desenvolvido usa a biblioteca $\mathrm{QT}^{2}$ e os algoritmos que definem cada um dos biótipos, conforme Lee et. al (2007). A biblioteca QT é uma biblioteca de funções, de código aberto, escrita em linguagem de programação ${\mathrm{C}++^{3}}^{3} \mathrm{e}$ extensível em Javascript ${ }^{4}$. A escolha por essa biblioteca de funções possibilitou, por exemplo, a instalação do sistema em dispositivo móvel (celular), o que vem a ser mais um facilitador durante o teste (realizado em campo, na segunda parte da pesquisa).

Figura 9 - Calculadora de biótipos com o resultado "Ampulheta Inferior"

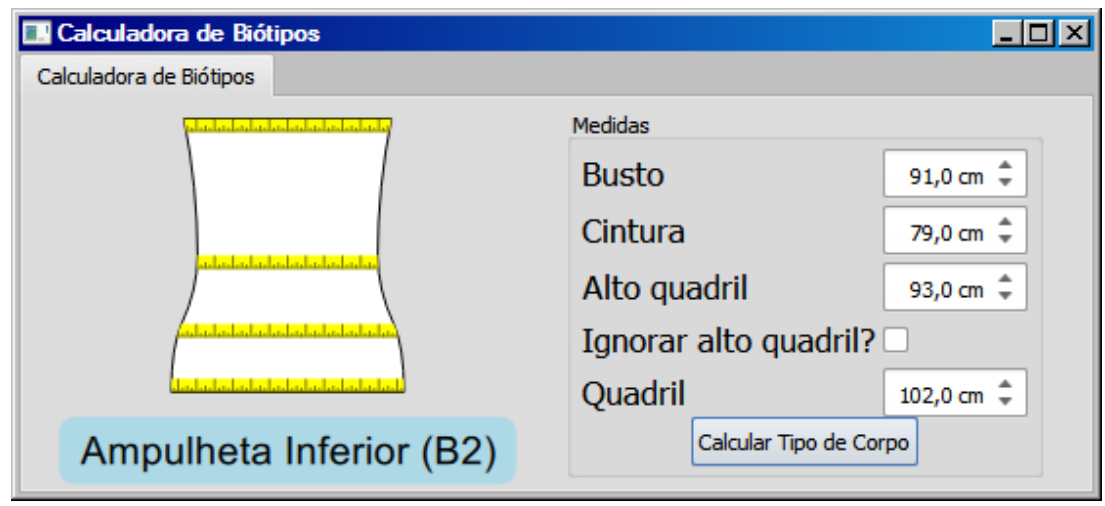

Fonte: Longhi e Smiderle (2016)

O software encontra-se disponível para utilização on-line, por meio do endereço eletrônico http://calcbio.paperplane.io/.

\section{Construção da TMA}

O desenvolvimento da tabela de medidas teve como ponto de partida as demais tabelas de medidas do vestuário utilizadas atualmente, especialmente àquela utilizada no Curso de Moda da UDESC.

Essa tabela foi elaborada com base em pesquisa dos professores do Curso de Moda que trabalham com a tecnologia do vestuário, sem comprometimento com nenhuma normalização,

\footnotetext{
${ }^{2}$ Biblioteca utilizada em programação para construção de interfaces.

${ }^{3}$ Linguagem de programação utilizada principalmente em programação para celular, desktop.

${ }^{4}$ Linguagem de programação utilizada principalmente em páginas da internet.
} 
sendo sua elaboração prevista apenas para o processo didático. Como seus valores são compatíveis com as demais tabelas de medidas pesquisadas no presente estudo, optou-se por verificar a qual biótipo pertenciam as medidas principais no tamanho 38 , ou seja, busto $84 \mathrm{~cm}$, cintura $64 \mathrm{~cm}$ e quadril $90 \mathrm{~cm}$. Assim, foi possível confrontar esses valores com o algoritmo de Lee at al. (2007) que define o biótipo ampulheta. Originalmente, os valores dos algoritmos estavam em polegadas, uma vez que os EUA não utilizam o sistema métrico de centímetros. Para fins didáticos, procedeu-se a substituição dos valores em polegadas pelos seus correspondentes em centímetros.

A seguir, partindo da confirmação de que os valores referentes às medidas de busto, cintura e quadril eram compatíveis com a fórmula do biótipo ampulheta, foi possível utilizar os valores da tabela de medidas da UDESC, e completar a primeira linha da TMA com os valores do biótipo ampulheta (B1). Os primeiros valores a serem preenchidos foram no tamanho 38/B1, no qual o busto mede $84 \mathrm{~cm}$, a cintura mede $64 \mathrm{~cm}$ e o quadril mede $90 \mathrm{~cm}$. Os demais valores referentes aos tamanhos 40,42, 44, 46, 48, 50 e 52, respeitaram uma progressão aritmética de 4 $\mathrm{cm}^{5}$. Por fim, procedeu-se ao preenchimento dos dados dos demais biótipos: ampulheta inferior, ampulheta superior, colher, triângulo, triângulo invertido e retângulo, conforme Lee et al. (2007).

\subsection{Escolha dos valores da TMA}

De acordo com Boueri (2010), no que concerne ao dimensionamento, cada situação requer que se utilize ora valores do limite superior, ora do limite inferior, e ora do limite médio. Isso varia conforme a necessidade de conforto, segurança, eficiência e conforme o público usuário de cada projeto. Segundo o autor, na elaboração de bonecos ou manequins para testes, deve-se produzir duas unidades, sendo uma com as medidas do limite inferior e outra com as do limite superior.

A definição dos números da tabela não seguiu um estrito critério em todos os biótipos. A questão principal era compor uma tabela de medidas na qual o valor de cada biótipo, além de um enquadramento matemático em função dos algoritmos, mantivesse alguma proporção. Em outras palavras, partindo do tamanho 38 , todas as combinações deveriam apresentar valores que fossem semelhantes em termos de dimensões (mantendo-se dentro do tamanho 38), mas variando em termos de formato do corpo. Essa era a principal demanda das tabelas de medidas estudas, que apresentavam uma progressão constante de quatro centímetros em cada tamanho, porém nunca apresentavam variação na forma. A TMA devia então apresentar variação na forma, porém respeitando um ponto de partida aproximado para cada biótipo.

Os estudos de Simmons, Stook e Devarajan (2004) apontam quais são as características físicas dos grupos femininos que caracterizam os biótipos e Lee et al. (2007) define as fórmulas matemáticas nas quais cada biótipo se encontra. Diante disso, foi preciso considerar quais eram os pontos anatômicos, nas medidas principais dos perímetros de busto, cintura e quadril, que apresentavam mais representatividade na definição de cada biótipo. Dessa forma, os biótipos ampulheta inferior, colher e triângulo, apresentavam um quadril mais destacado, já nos biótipos ampulheta superior, e triângulo invertido, o destaque era a região do busto.

De posse de todas essas informações e dispondo do software para cálculo de biótipos,

\footnotetext{
${ }^{5}$ A tabela de medidas da UDESC aplica uma progressão de $6 \mathrm{~cm}$ a partir do tamanho 46 . Essa variação na progressão naqueles tamanhos considerados plus size (tamanhos maiores) não foi mantida no presente estudo por não ser esse o escopo da pesquisa e por tratar-se de uma questão que merece um estudo mais aprofundado.
} 
procedeu-se ao teste das combinações que iriam compor a TMA. O tamanho 38 já definido pelo biótipo ampulheta (B1), balizou a escolha das medidas dos demais biótipos no mesmo tamanho.

Após a definição do tamanho 38 de cada biótipo, partiu-se para o preenchimento dos demais tamanhos da tabela, numa progressão aritmética de quatro centímetros. Entretanto, no biótipo colher (B4), do tamanho 46 em diante, a progressão é de cinco centímetros.

As demais medidas da tabela podem ser consideradas medidas auxiliares, são elas: largura das costas, altura do busto, altura da cintura, altura do quadril e degolo. Estas medidas foram preenchidas no intuito de fornecer os dados mínimos necessários para o desenho do diagrama de modelagem de vestuário de cada biótipo. Optou-se então por utilizar valores padronizados para todos os biótipos, uma vez que o escopo do trabalho se resume a avaliação dos perímetros das medidas principais - busto, cintura e quadril.

\section{Resultados}

O resultado obtido foi a Tabela de Medidas Associáveis (TMA), que permite o desenvolvimento de bases para mais de um tipo físico, sem a necessidade de consulta de outras fontes, conforme Figura 10.

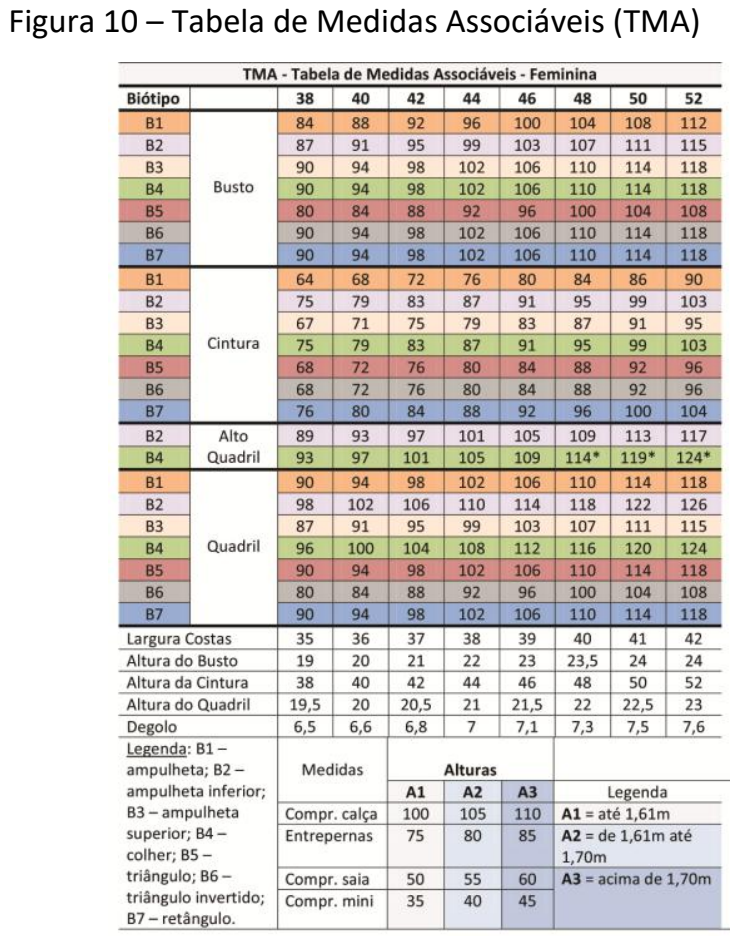

Fonte: os autores

Com o objetivo de facilitar a localização e a diferenciação entre as faixas de valores, foram atribuídas cores diferentes para cada biótipo. Também foi definida a sigla B acrescida de um número de um a sete, assim, tem-se: B1 - biótipo ampulheta; B2 - biótipo ampulheta inferior; B3 biótipo ampulheta superior; B4 - biótipo colher; B5 - biótipo triângulo; B6 - biótipo triângulo invertido; B7 - biótipo retângulo. 
No que concerne às estaturas, foram apresentadas três alturas principais: A1 para pessoas com altura de até $161 \mathrm{~cm}$; A2 para pessoas com altura entre $161 \mathrm{~cm}$ e $170 \mathrm{~cm}$; A3 para pessoas com mais de $170 \mathrm{~cm}$. Esses valores são compatíveis com as faixas de altura encontradas na pesquisa SizeBR, segundo Bastos et al. (2013). Essas medidas são úteis para a construção de calças com variados comprimentos de perna - curta, média e longa

Ao consultar os valores da TMA foi possível desenhar o diagrama de cada um dos biótipos, a partir do qual são feitos os moldes de vestuário. Diante de cada um dos diagramas, foi visível a diferença dos formatos de cada um dos tipos físicos, ainda que somente em duas dimensões. Testes de ajuste e caimento de vestuário se fazem necessários para a comprovação da relação entre os biótipos e a forma do vestuário. Independentemente da sua utilização prática, a TMA pode ser utilizada satisfatoriamente.

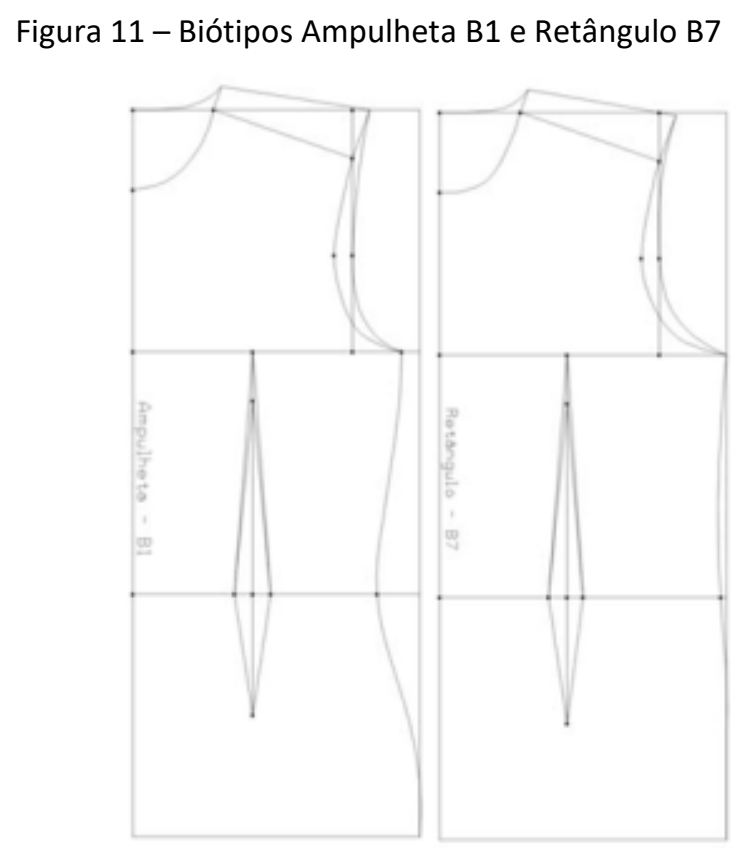

Fonte: os autores

A figura 11 apresenta dois diagramas feitos com base nos valores da TMA, referentes ao biótipo ampulheta e ao biótipo retângulo. É visível a diferença nas linhas de contorno do corpo, na qual observa-se a cintura marcada no biótipo ampulheta e quase reta no retângulo. A aplicação prática da TMA reside na possibilidade de escolha de qual biótipo adotar como referência de corpo para o desenvolvimento da modelagem. A calculadora de Biótipos (Calcbio) por sua vez, serve como uma ferramenta de captura e cálculo de medidas das usuárias. O conhecimento dos variados biótipos e o uso destes instrumentos possibilita o desenvolvimento de peças de vestuário capazes de trazer mais conforto e satisfação às usuárias.

\section{Conclusões}

O trabalho na indústria do vestuário, bem como em qualquer ramo industrial, deve ser ágil e preciso, de modo a evitar retrabalho e perdas financeiras. Na outra ponta da cadeia está o 
usuário que consome esse vestuário industrializado, muitas vezes feito de modo padronizado, sem espaço para a variedade antropométrica inerente ao ser humano.

O conhecimento dos biótipos femininos pode contribuir para o avanço na construção de um vestuário mais ergonômico, além de trazer mais satisfação aos usuários. Também pode acarretar uma economia de matéria-prima, uma vez que a indústria pode mensurar o real tamanho e formato dos usuários das peças produzidas.

Para a academia, esse conhecimento permite aos futuros profissionais projetarem produtos baseados em seres humanos reais e não idealizados, sem prejuízo do processo criativo. Embora o vestuário pronto possa variar em função do modelo e da matéria-prima, é importante desenvolver uma base de modelagem o mais próxima possível da forma do usuário final.

No intuito de propor uma abordagem do tema com aplicabilidade na prática do desenvolvimento de vestuário, foi desenvolvida a Tabela de Medidas Associáveis - TMA, na qual são apresentados os sete biótipos estudados. A proposta foi desenvolver uma ferramenta que permitisse ao modelista visualizar simultaneamente algumas das principais combinações em termos de formas e tamanhos de corpos femininos, incluindo variações de estatura.

O software de verificação de biótipos (Calculadora de biótipos) foi desenvolvido para permitir o correto preenchimento da tabela de medidas e posterior teste com usuárias. Porém, também pode vir a ser utilizado como instrumento de coleta de dados por parte de outros pesquisadores e de empresas, ao possibilitar a criação de um banco de dados de medidas e formas físicas das suas clientes. Posteriormente, o setor de modelagem pode desenvolver peças piloto com os biótipos mais comumente encontrados entre as usuárias e obter um melhor caimento das peças e, consequentemente, mais satisfação do público.

A TMA é uma tabela de medidas associáveis porque permite que o modelista associe medidas do perímetro com medidas de estatura. Também permite que cada empresa monte a sua tabela de medidas de acordo com os tipos físicos mais frequentes entre os usuários dos seus produtos. E finalmente, o principal objetivo do estudo foi demonstrar a viabilidade do trabalho de desenvolvimento de modelagem do vestuário, considerando a variedade de formas e estaturas do público feminino brasileiro.

\section{Referências}

ASSOCIAÇÃO BRASILEIRA DE NORMAS TÉCNICAS. NBR 13377: medidas do corpo humano para vestuário - padrões referenciais. In: ABNT Catálogo, 1995. (http://www.abntcatalogo.com.br).

BASTOS, Sergio F. et al. SizeBR - O Estudo Antropométrico Brasileiro. In: 4th International Conference and Exhibition on 3D Body Scanning Technologies. Long Beach/CA, 2013. (http://www.portaldaindustria.com.br).

BASTOS, Sergio F.; SABRÁ, Flávio. A forma do corpo da mulher brasileira. In: 5th International Conference and Exhibition on 3D Body Scanning Technologies. Lugano/Switzerland, 2014. (http://www.portaldaindustria.com.br).

BOUERI, José J. Antropometria Aplicada ao Projeto e Dimensionamento do Vestuário Brasileiro. São Paulo: 2010. Relatório Científico Final Projeto FAPESP. 
DINIS, Patrícia M.; VASCONCELOS, Amanda F. C. Modelagem. In: SABRÁ, Flávio (Org.). Modelagem: Tecnologia em produção de vestuário. São Paulo: Estação das Letras e Cores, 2014. p. 54-125.

KEISER, Sandra J.; GARNER, Myrna B. Beyond design: the synergy of apparel product development. 2nd ed. New York: Fairchild Publications, 2008.

LEE, J.Y. et al. Comparison of body shape between USA and Korean women. International Journal of Clothing Science and Technology. Vol. 19, No. 5, pp. 374-391, 2007.

LONGHI, Tatiana Castro. Critérios ergonômicos no vestuário: uma proposta de sistematização de dados antropométricos. 128 p. Dissertação (Mestrado) - Universidade do Estado de Santa Catarina, Centro de Artes, Mestrado em Design, Florianópolis, 2017.

SILVEIRA, Icléia. Apostila de Modelagem Básica do Vestuário Feminino. Curso de Bacharelado em Moda - Habilitação em Design de Moda. Universidade do Estado de Santa Catarina - UDESC / CEART, Florianópolis, 2015.

SIMMONS, Karla; ISTOOK, Cynthia L.; DEVARAJAN, Priya. Female Figure Identification Technique (FFIT) for Apparel. Part I: Describing Female Shapes. Journal of Textile and Apparel, Technology and Management, Vol. 4, Issue 1, 2004. 